\title{
Study Protocol: Using Deep- Brain Stimulation, Multimodal Neuroimaging and Neuroethics to Understand and Treat Severe Enduring Anorexia Nervosa
}

\author{
Rebecca J. Park', Jessica C. Scaife ${ }^{1 *}$ and Tipu Z. Aziz ${ }^{2}$ \\ 'Department of Psychiatry, Warneford Hospital, University of Oxford, Oxford Health NHS Foundation Trust, Oxford, \\ United Kingdom, ${ }^{2}$ Nuffield Department of Clinical Neurosciences, University of Oxford, Oxford, United Kingdom
}

OPEN ACCESS

Edited by:

Paul Croarkin,

Mayo Clinic Minnesota,

United States

Reviewed by:

Berthold Langguth,

University of Regensburg,

Germany

Francesco Sammartino,

The Ohio State University,

United States

Veljko Dubljevic,

North Carolina State

University, United States

*Correspondence:

Jessica C. Scaife

jessica.scaife@psych.ox.ac.uk

Specialty section:

This article was submitted to Neuroimaging and Stimulation,

a section of the journal

Frontiers in Psychiatry

Received: 03 October 2017 Accepted: 22 January 2018

Published: 06 April 2018

Citation:

Park RJ, Scaife JC and Aziz TZ

(2018) Study Protocol: Using Deep-Brain Stimulation, Multimodal

Neuroimaging and Neuroethics

to Understand and Treat Severe

Enduring Anorexia Nervosa.

Front. Psychiatry 9:24.

doi: 10.3389/fpsyt.2018.00024
Background: Research suggests that altered eating and the pursuit of thinness in anorexia nervosa (AN) are, in part, a consequence of aberrant reward circuitry. The neural circuits involved in reward processing and compulsivity overlap significantly, and this has been suggested as a transdiagnostic factor underpinning obsessive compulsive disorder, addictions and eating disorders. The nucleus accumbens (NAcc) is central to both reward processing and compulsivity. In previous studies, deep-brain stimulation (DBS) to the NAcc has been shown to result in neural and symptomatic improvement in both obsessive compulsive disorder and addictions. Moreover, in rats, DBS to the NAcc medial shell increases food intake. We hypothesise that this treatment may be of benefit in severe and enduring anorexia nervosa (SE-AN), but first, feasibility and ethical standards need to be established. The aims of this study are as follows: (1) to provide feasibility and preliminary efficacy data on DBS to the NAcc as a treatment for SE-AN; (2) to assess any subsequent neural changes and (3) to develop a neuroethical gold standard to guide applications of this treatment.

Method: This is a longitudinal study of six individuals with SE-AN of $>7$ years. It includes an integrated neuroethical sub-study. DBS will be applied to the NAcc and we will track the mechanisms underpinning AN using magnetoelectroencephalography, neuropsychological and behavioural measures. Serial measures will be taken on each intensively studied patient, pre- and post-DBS system insertion. This will allow elucidation of the processes involved in symptomatic change over a 15-month period, which includes a double-blind crossover phase of stimulator on/off.

Discussion: Novel, empirical treatments for SE-AN are urgently required due to high morbidity and mortality costs. If feasible and effective, DBS to the NAcc could be game-changing in the management of this condition. A neuroethical gold standard is crucial to optimally underpin such treatment development.

Clinical Trial Registration: The study is ongoing and registered with www.ClinicalTrials. gov, https://clinicaltrials.gov/ct2/show/NCT01924598, 22 July, 2013. It has full ethical and HRA approval (Project ID 128658).

Keywords: anorexia nervosa, treatment, clinical trial, compulsivity, reward, deep-brain stimulation 


\section{INTRODUCTION}

Anorexia nervosa (AN) is one of the most challenging psychiatric disorders to treat, and its mortality rate is the highest amongst the psychiatric disorders $(1,2)$. It becomes severe and enduring anorexia nervosa (SE-AN) in at least a third of cases. There remains a grave paucity of evidence-based psychological therapies for AN (3-5), and there are few psychopharmacological treatments of benefit (6-8). The lack of effective treatments for SE-AN leads to huge morbidity costs to individuals and healthcare services (9), so the development of novel, empirically-based and effective treatments is of major importance. This study investigates whether deep-brain stimulation (DBS) of the ventral anterior limb of the internal capsule (ALIC) within the nucleus accumbens (NAcc) is acceptable, feasible and helpful for adults with SE-AN.

DBS is a reversible, adjustable, non-destructive intervention using a surgically implanted medical device, which delivers carefully controlled electrical pulses to precisely targeted brain areas. While the exact mechanisms of action remain unclear, it has been suggested that DBS works by rebalancing restingstate networks (RSNs) in the brain. Neuroimaging studies have demonstrated that RSNs are abnormal in AN (10-12). In this study, we will use magnetoencephalography (MEG) to monitor post-operative changes in neural activity. Recordings will be made both in the resting state and also during a food reward task in which participants are asked how much they want high- vs low-calorie food stimuli. MEG scanning is a real-time measure of neural responses, which we have previously used to demonstrate attentional bias to food cues in a currently ill population (13).

DBS has been widely used, particularly for Parkinson's disease, for over 30 years. Increasingly over the last decade, DBS has been applied to the treatment of psychiatric disorders including treatment-resistant depression (14-17), obsessive compulsive disorder (OCD) $(18,19)$ and addictions $(20)$. The US Food and Drug Administration approved a humanitarian device exemption for DBS in the treatment of severe OCD in 2009. This resulted from a review of data from 26 patients with severe treatment-resistant OCD who underwent DBS to the ALIC/ventral striatum (VS). On average, after 12 months of therapy, there was a $40 \%$ reduction in patients' OCD symptoms (21).

Two lines of evidence indicate that DBS might be effective in SE-AN. Firstly, OCD and AN are highly co-morbid, with both disorders showing a high degree of compulsivity (22-24). The

Abbreviations: ALIC, Anterior Limb Internal Capsule; AN, Anorexia Nervosa; BMI, Body Mass Index; CANTAB, Cambridge Neuropsychological Test Automated Battery; CI, Chief Investigator; CTRG, Clinical Trials \& Research Governance, University of Oxford; DBS, Deep-brain stimulation; DTI, Diffusion Tensor Imaging; FMRIB, fMRI Brain Imaging Centre, Oxford (John Radcliffe Hospital); HRA, Health Research Authority; IRAS, The Integrated Research Application System; NAcc, Nucleus Accumbens; NRES, National Research Ethics Service; OHBA, Oxford Centre for Human Brain Activity; PI, Principal Investigator; PIS, Patient Information Sheet; R\&D, NHS Trust R\&D Department; REC, Research Ethics Committee; RSN, Resting-State Network; SAE, Serious Adverse Event; SE-AN, Severe and Enduring Anorexia Nervosa; VS, Ventral Striatum. second arises from case reports and series. A patient with severe OCD also found improvement in co-morbid anorexia following DBS (25). Further case reports and case series of DBS for SE-AN (26-28) suggest benefit, although none have included doubleblind DBS on-off phases. The largest case series, of 6 patients with SE-AN (29) involved DBS applied to the subcallosal cingulate, a neural target previously used for treatment-resistant depression. Regarding safety, the authors initially reported one serious adverse event (SAE): a seizure 2 weeks post-operatively in the context of AN-related metabolic disturbance, with no permanent sequelae. A further patient had a panic attack intraoperatively, and another had an air embolism, but there were no long-lasting adverse effects of the surgery for either participant. A follow-up paper reported sustained symptomatic improvement and weight restoration in three out of six patients, possibly mediated by improvements in mood regulation (30). A recent single case study has also reported improvement in eating disorder psychopathology and mood in an individual with chronic AN, following DBS to the bed nucleus of the stria terminalis (28).

Individuals with $\mathrm{AN}$ experience an aberrant sense of reward from weight loss and self-starvation $(9,31,32)$ with neural evidence that reward processing becomes abnormal $(33,34)$. Energy-dense food is anxiety-provoking and aversive, such that individuals become unable to eat to sustain life $(35,36)$. The compulsive pursuit of self-starvation in AN represents a major barrier to treatability, rendering contemporary treatments highly aversive and contributing to high dropout and relapse rates $(31,37)$. If these disturbed reward processes could be characterised and subsequently interrupted at a neural level, it would revolutionise the treatment of $\mathrm{AN}$.

The NAcc, located deep in the VS, is central to reward processing and has been described as a "hedonic hotspot" [38]. In rats, DBS to the medial shell of the NAcc has been shown to increase food intake (39), and activity in this structure is key to experiencing food as rewarding (38). Neuroimaging and experimental studies in $\mathrm{AN}(31,32,37)$ confirm a dysregulation of reward circuitry: in particular, an abnormal striatal response to food and thinness cues $(33,34,40)$. There is also evidence of an increased top-down control over reward circuitry in $\mathrm{AN}$, emanating from the prefrontal cortex (41).

The relentless self-starvation and over-exercising seen in AN have parallels with OCD and addictions with evidence of aberrant reward processing in common with these other pathologies $(22,24,42-45)$. There is evidence that compulsivity is a transdiagnostic process common across these disorders (23), mediated at a neural level by corticostriatal thalamic circuits, which incorporates the VA and NAcc (46). Excessive reliance on habit formation in learning has been suggested as the basis of persistent compulsive behaviour in OCD (47) and more recently in $\mathrm{AN}$ (9). It has been suggested that restrictive eating and weight loss in AN begin as "action-outcome/goal-directed learning" in which behaviour is associated with a rewarding outcome (44). However, through repetition, reward processing becomes aberrant such that the behaviour no longer relies upon reward reinforcement (stimulus-response learning), and this behaviour becomes highly resistant to change (48). 
This reduction in goal-directed learning in eating disorders has been demonstrated using a two-step habit formation task (49). This task will also be undertaken by participants in this study to record any changes in compulsive, habitual responding (50).

Given the high mortality and morbidity rates of SE-AN (51), additional risks to patients of surgery and entering this study are small, with a potential cost-benefit analysis that justifies ethical equipoise (52). Professor Tipu Aziz, who performs all the DBS operations, has an excellent personal safety record and has performed thousands of operation on patients with Parkinson's disease. He has also operated on adults with chronic pain and children with movement disorders. The procedure itself has an overall risk of stroke of less than $1 \%$, a haemorrhage-causing death of $0.01 \%$ and a risk of wound infection of $5 \%$. To minimise the risk of surgery, the patients will be medically stable prior to surgery. They must have a normal ECG and electrolytes, be free of severe binging or purging or severe depression/suicidality, both of which pose added background risks. The technique (as performed in Oxford) is very well tolerated and can be done in one stage, supported by two to four nights as an inpatient in the neurosurgical ward. DBS has a one-off cost of approxi-

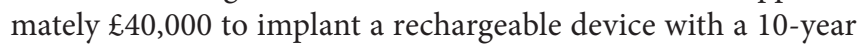
lifespan. As the mechanisms, efficacy and optimal DBS target for intervention in AN are yet to be established, it should be regarded as an experimental treatment, with the risk that it will not be effective. However, given the extremely poor outcome in terms of morbidity and mortality for those with SE-AN, it is plausibly an intervention where the benefits may outweigh the risks and costs.

A significant body of research suggests that altered reward processing contributes to the maintenance of SE-AN and that this is influenced by higher cognitive control processes (9). We hypothesise that DBS to the NAcc may ameliorate aberrant reward processing and AN psychopathology.

In this clinical trial, our aims are to examine (1) the safety, acceptability and feasibility of DBS to the ALIC at the NAcc in SE-AN; (2) to map neural mechanisms and symptomatic change following DBS; (3) to explore ethical issues, capacity, consent and patients' views pre- and post DBS and (4) to explore the longerterm effects of DBS in SE-AN.

\section{METHODS}

This is a study of DBS targeting the NAcc in up to six patients with SE-AN, using pre- and post-operative repeated measures. To date, five patients have been enrolled in the study.

The study period is 15 months-see Figure $\mathbf{1}$ for details of study interventions. There will be pre-operative assessments in month 1 , the DBS operation in month 2, followed by the DBS switch-on at the end of month 3. DBS dose will be optimised during months $3-6$. During month 10 of the study (6 months

\begin{tabular}{|c|c|c|c|c|c|c|c|c|c|c|c|c|c|c|c|c|c|}
\hline \multirow{4}{*}{$\begin{array}{l}\text { Study Procedures } \\
\text { Month in study }\end{array}$} & \multicolumn{17}{|c|}{ Phase of study: monthly visits except month $3-5$, month $10^{*}$} \\
\hline & \multirow{3}{*}{$\begin{array}{l} \\
\\
-1\end{array}$} & \multirow{3}{*}{ 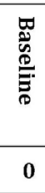 } & \multirow{3}{*}{ 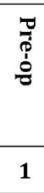 } & \multirow{3}{*}{ 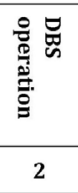 } & switch & \multicolumn{6}{|c|}{ Post switch on } & \multirow{3}{*}{$\begin{array}{l}\begin{array}{l}2 \times 2 \mathrm{w} \\
\text { Double } \\
\text { blind on/ off } \\
\text { period }\end{array} \\
10 \\
\end{array}$} & \multirow{2}{*}{\multicolumn{5}{|c|}{ Dose stabilization period (DBS ON) }} \\
\hline & & & & & \multicolumn{3}{|c|}{$\begin{array}{l}\text { Dose optimisation } \\
\text { period }\end{array}$} & \multicolumn{4}{|c|}{$\begin{array}{l}\text { Dose stabilization } \\
\text { Period (DBS ON) }\end{array}$} & & & & & & \\
\hline & & & & & 3 & 4 & 5 & 6 & 7 & 8 & 9 & & 11 & 12 & 13 & 14 & 15 \\
\hline Informed consent & & $\mathrm{x}$ & & & & & & & & & & & & & & & \\
\hline Ethical assessment & & $\mathrm{x}$ & & & & & & & & & & & & & & & \\
\hline $\begin{array}{l}\text { Medical \& } \\
\text { psychiatric } \\
\text { history }\end{array}$ & $\mathrm{x}$ & & & & & & & & & & & & & & & & \\
\hline $\begin{array}{l}\text { Psychiatric /ED } \\
\text { assessment, BMI }\end{array}$ & $\mathrm{x}$ & $\mathrm{x}$ & $\mathrm{x}$ & $\mathrm{x}$ & $\mathrm{x}$ & $\mathrm{x}$ & $\mathrm{x}$ & $\mathrm{x}$ & $\mathrm{x}$ & $\mathrm{x}$ & $\mathrm{x}$ & $\mathrm{x} 2$ & $\mathrm{x}$ & $\mathrm{x}$ & $\mathrm{x}$ & $\mathrm{x}$ & $\mathrm{x}$ \\
\hline ECG and bloods & & & $\mathrm{x}$ & $\mathrm{x}$ & & & & & & & & & & & & & \\
\hline MRI/CT & & & $\mathrm{x}$ & $\mathrm{x}$ & & & & & & & & & & & & & \\
\hline $\begin{array}{l}\text { Neurosurgical } \\
\text { review }\end{array}$ & & $\mathrm{x}$ & $\mathrm{x}$ & $\mathrm{x}$ & $\mathrm{x}$ & $\mathrm{x}$ & $\mathrm{x}$ & $\mathrm{x}$ & $\mathrm{x}$ & $\mathrm{x}$ & $\mathrm{x}$ & $\mathrm{x} 2$ & $\mathrm{x}$ & $\mathrm{x}$ & $\mathrm{x}$ & $\mathrm{x}$ & $\mathrm{x}$ \\
\hline $\begin{array}{l}\text { Neuropsychological } \\
\text { battery }\end{array}$ & & $\mathrm{x}$ & & & & & & & & & & & & & & & $\mathrm{x}$ \\
\hline Self-report schedules & & $\mathrm{x}$ & $\mathrm{x}$ & $\mathrm{x}$ & $\mathrm{x}$ & $\mathrm{x}$ & $\mathrm{x}$ & $\mathrm{x}$ & $\mathrm{x}$ & $\mathrm{x}$ & $\mathrm{x}$ & $\mathrm{x} 2$ & $\mathrm{x}$ & $\mathrm{x}$ & $\mathrm{x}$ & $\mathrm{x}$ & $\mathrm{x}$ \\
\hline Habit \& CANTAB tasks & & $\mathrm{x}$ & & & & & & & & & & $\mathrm{x} 2$ & & & & & $\mathrm{x}$ \\
\hline MEG scan & & $\mathrm{x}$ & & & $\mathrm{x}$ & & & & & & & $\mathrm{x} 2$ & & & & & $\mathrm{x}$ \\
\hline $\begin{array}{l}\text { Food Preference } \\
\text { Task }\end{array}$ & & $\mathrm{x}$ & & & $\mathrm{x}$ & & & & & & & $\mathrm{x} 2$ & & & & & $\mathrm{x}$ \\
\hline $\begin{array}{l}\text { Adverse events \& } \\
\text { side effects assessed }\end{array}$ & & & & $\mathrm{x}$ & $\mathrm{x}$ & $\mathrm{x}$ & $\mathrm{x}$ & $\mathrm{x}$ & $\mathrm{x}$ & $\mathrm{x}$ & $\mathrm{x}$ & $\mathrm{x}$ & $\mathrm{x}$ & $\mathrm{x}$ & $\mathrm{x}$ & $\mathrm{x}$ & $\mathrm{x}$ \\
\hline $\begin{array}{l}1-2 \text { weekly dose } \\
\text { *adjustments }\end{array}$ & & & & & $\mathrm{x}$ & $\mathrm{x}$ & $\mathrm{x}$ & & & & & $\mathrm{x}$ & & & & & \\
\hline On line 1-1 support & & $\mathrm{x}$ & $\mathrm{x}$ & $\mathrm{x}$ & $\mathrm{x}$ & $\mathrm{x}$ & $\mathrm{x}$ & $\mathrm{x}$ & $\mathrm{x}$ & $\mathrm{x}$ & $\mathrm{x}$ & $\mathrm{x}$ & $\mathrm{x}$ & $\mathrm{x}$ & $\mathrm{x}$ & $\mathrm{x}$ & $\mathrm{x}$ \\
\hline
\end{tabular}

FIGURE 1 | Schedule of study procedures. 
after switch-on), there will be two double-blinded 2-week periods of DBS on/off as described by Denys et al. (53). At 15 months (12 months after switch-on), there will be final follow-up tests. This study is a quantitative and qualitative investigation of eating disorder and co-morbid pathology, using MEG to image neural processes, and computerised tasks to assess habit formation. There is also a parallel quantitative and qualitative sub-study of ethical issues involved.

\section{Patient Population and Recruitment Inclusion Criteria}

- Primary diagnosis: AN according to the DSM-V criteria, based on a psychiatric interview

- Illness duration of severe AN of $>7$ years

- Disabling severity with substantial functional impairment

- Treatment refractoriness, defined as lack of response to two or more typical modes of treatment, such as inpatient weight restoration, psychotherapy and psychopharmacology

- Severely underweight: Body Mass Index (BMI) $>13<16$

- 21-65 years old

- Written informed consent

- Able to fully understand the consequences of the procedure

- English speaking and able to answer the study questions fluently

- Has the mental capacity to provide informed consent to research participation

\section{Exclusion Criteria}

- Unstable physical condition (severe electrolyte disturbances, cardiac failure and other physical conditions due to low weight in which surgery/anaesthesia is contraindicated)

- Treatable underlying cause of anorexia/underweight

- Parkinson's disease, dementia, epilepsy

- History of schizophrenia/psychosis, bipolar disorder

- Alcohol or substance abuse (including benzodiazepines) during the last 6 months

- Current severe major depressive or Tic disorder

- Antisocial or severe Borderline Personality Disorder

- Standard MRI scan exclusion criteria (pregnancy, pacemaker and metals contraindicated for MRI, except for the DBS implantation and stimulator itself)

- Currently an involuntary patient

\section{Patient Selection Process}

The study patients will have SE-AN. Individuals fulfilling inclusion and exclusion criteria under the clinical care of specialist eating disorder services will be ranked in the order of suitability according to the below specific criteria:

- Degree of severity and intractability of AN

- Has never had periods of full remission

- Has tried existing treatment options without success

- Intensity of desire to recover

- Restrictive AN-without bingeing/purging

- Absence of currently severe co-morbid depression or self-harm

- Ideally on no, or minimal, psychotropic medication
- No recent history of compulsory treatment (within the last 12 months)

- Good intellectual function and education-well able to comprehend the facts of the intervention

- Poor social and occupational function, high levels of distressnot functioning well, has poor quality of life

A ranked list of potential participants will be made in consultation with direct clinical care consultants prior to Rebecca Park approaching patient(s) one by one in rank order. If the first is not suitable or not interested, the next ranked will be approached.

\section{TRIAL SITES}

The study involves collaboration between the Department of Psychiatry and the Department of Surgery at the University of Oxford. Research activities will take place at the Department of Psychiatry, University of Oxford, Warneford Hospital and the Department of Neurosurgery, John Radcliffe Hospital, Oxford.

\section{Phases of the Study}

\section{Phase I: Screening and Enrolment}

Suitable patients will be identified in close liaison with specialist eating disorders clinical teams who can consult Rebecca Park initially as to their suitability. If felt to be potentially suitable, the patient will first be informed of the opportunity to take part in the study by their clinical care team. If they show interest, they will be provided with a patient information sheet (PIS) and will meet with Rebecca Park initially for a preliminary assessment. If deemed suitable, the patient will then meet Rebecca Park and Tipu Aziz jointly for further information, either alone or with their family, with ample opportunity for questions. They will be given at least a week to decide whether to participate, before being consented by Rebecca Park. At all times, participants will be assured that their participation is entirely voluntary and that they may withdraw from the study at any point with no implications for their clinical care.

All patients will need to have normal electrolytes, and ECG and anaesthetic review prior to being considered fit for the operation. Patients need to have a BMI of over 13 for surgery.

\section{Phase II: Pre-operative Baseline Evaluation Assessment with Research Team (Repeated at All Subsequent Monthly Follow-ups)}

Semi-structured interviews assessing psychiatric symptoms (including eating disorder symptoms) will be performed, along with the completion of self-report questionnaires which index eating disorder psychopathology, mood and participants' quality of life. This is a battery of validated standardised questionnaires, of gold-standard use in studies of eating disorders and OCD, which will be used to assess mood, anxiety, obsessionality and eating disorder psychopathology:

- Eating Disorder Examination (EDE) (54)

- Global Assessment of Functioning DSM-IV (55)

- Structured Clinical Interview for DSM-5 (56)

- Hamilton Anxiety Rating Scale (57) 
- Hamilton Depression Response Scale (58)

- Yale-Brown-Obsessive Compulsive Scale and checklist (59)

- Yale-Brown-Cornell Eating Disorder Scale (60)

- EDE Questionnaire (61)

- Self-Starvation Scale (62)

- Clinical Impairment Assessment (63)

- WHO Quality of Life Scale (64)

- Beck Depression Inventory (65)

- State Trait Anxiety Inventory (66)

- Snaith-Hamilton Pleasure Scale (67)

- Ruminative Response Scale for Eating Disorders (68)

- BMI is also calculated during the baseline assessment.

\section{Neuropsychological Assessment}

A neuropsychological assessment battery will be carried out by a clinical neuropsychologist. This comprises the following:

- WAIS-IV subtests: Vocabulary, Matrix Reasoning, Digit Span, Coding, Similarities (69)

- BIRT Memory and Information Processing Battery: ListLearning Task (70)

- Verbal Fluency (Baseline Measure of Phonemic and Semantic Fluency) (71)

- Rey Complex Figure task-assessment of the ability to copy, immediate recall, delayed recall (72)

- Iowa Gambling Task (73)

- Trail-Making Task Parts A \& B (74)

- D-KEFS Colour Word Interference (75)

- Hospital Anxiety and Depression Scale (76).

Pre-operative Capacity Assessment and Ethical Sub-Study

Once each suitable patient is identified and consented, then Jacinta Tan, an independent psychiatrist and ethicist, will interview the patient and perform a complete MacCAT-CR assessment (77), along with an in-depth interview to explore the patient's experience of treatment, rationale for participation and motivations for taking part in research. This follows the Oxford Neuroethics Research Paradigm which ensures a high level of capacity, voluntariness and informed consent. It is described in more detail in our recently published paper (52) and also on the registered trial online: https://clinicaltrials. gov/show/NCT01924598. We integrated this ethical sub-study from the inception of this protocol as recommended by recent consensus guidelines (78). It is guided by the foundational principles of the Nuffield Council of Bioethics report on "Intervening in the Brain" (79).

The assessment will take $2.5-3 \mathrm{~h}$ and can be done in sections if the patient feels tired. It will be tape-recorded and transcribed. Jacinta Tan and an independent observer will separately score the MacCAT-CR. Both will also provide global assessments of capacity to consent to research. Jacinta Tan will provide a detailed report of the interview and her assessment of ethical acceptability of participation for that individual. This will ensure that the needs of the research are not prioritised over those of the patient. If the patient is not suitable, the process will be repeated with the next interested and potentially suitable individual on the list.

\section{Neuroimaging and Computer Tasks}

Pre-operatively, patients will undergo the following:

- A whole-brain MRI scan including T1, T2 and Inversion Recovery sequences at the John Radcliffe Hospital, Oxford, with a total duration of $20 \mathrm{~min}$.

- A whole-brain MEG scan: a resting state and a food-"wanting" task (13), which will be performed at OHBA, the Warneford Hospital, Oxford, with a duration of $20 \mathrm{~min}$.

- Computerised behavioural tasks of reward: The Leeds Oxford Food Preference Questionnaire (LOFPQ) (35), 2-step habit formation task (50) and tasks from the Cambridge Neuropsychological Test Automated Battery (CANTAB) (80).

\section{True Colours}

Participants will complete weekly self-report symptom questionnaires at home (estimated to be $5 \mathrm{~min}$ ) using the "True Colours" online self-monitoring system pioneered at the University of Oxford, Department of Psychiatry, adapted for Eating Disorders by Rebecca Park by including the OxBREaD brief-rating scale: http://oxtext.psych.ox.ac.uk/true-colours. This self-report system will continue from the study entry to the study end.

\section{Phase III Operative Phase: Month 2}

The intervention consists of bilateral DBS targeted at the NAcc with stimulation at the ventral ALIC. The stimulation can be programmed and adjusted non-invasively by a trained clinician to minimise side effects and maximise symptom control.

The DBS system consists of three components: two electrode leads that are inserted into the brain, the implanted pulse generator (IPG) that contains a battery and a circuitry to produce the stimulus current, and the extension leads that run subcutaneously to connect these together. All three components are surgically implanted inside the body, with the IPG typically placed subcutaneously in the pectoral or the abdominal region. Patients will be implanted with the Medtronic RC pacemaker and the 3387 electrode. After implantation, the IPG can be calibrated by a trained clinician to optimise symptom suppression and control side effects. A rechargeable pacemaker lasts for 10 years and is much smaller than a non-rechargeable one, so is ideal for those who are underweight.

The whole procedure will be completed in 1 day and in total will require up to 4 days and 3 nights of inpatient stay at the John Radcliffe Hospital. On the day of surgery, the patient will be anaesthetised and the base ring fixed to the skull and a CT scan performed with a localiser fixed to the ring. The patient will then be transferred to theatre and the scalp cleansed. By fusing the structural MRI scan to the stereotactic CT scan, the trajectory to implant electrodes into the NAcc bilaterally will be calculated. Then, through bilateral scalp incisions and a twist-drill skull perforation ( $2.5 \mathrm{~mm}$ in diameter), deep-brain electrodes will be passed to target bilaterally and fixed to the skull with titanium mini plates. The scalp will then be closed, and a repeat stereotactic CT scan will be obtained to confirm electrode placement.

Having done so, back in the theatre, the electrodes will be connected to extension cables that will then be passed subcutaneously down one side of the head, behind the ear to 
a subcutaneous pouch below the collar bone and connected to a rechargeable pacemaker and all the wounds closed. Video recordings are used as part of the assessment process during routine implantation of DBS wires, whether taking part in a study or not, and are therefore considered part of standard operating procedure.

The day after surgery, the pacemaker will be turned on to ascertain any immediate effects of stimulation on symptoms and then switched off again. The patient will then be discharged 2 days after implantation. The stitches will be removed and the wound checked after 2 weeks. They will be assessed a month later when all wounds are healed and any acute effects of the DBS electrode insertion operation such as headaches, scalp soreness or wound tenderness have worn off. If so, DBS will be switched on: patients will be turned on to a setting of deepest contact -ve, third contact +ve, amplitude $2.5 \mathrm{~V}$, pulse width $60 \mathrm{~ms}$ and frequency $130 \mathrm{~Hz}$. Patients will have monthly assessments, and by the third visit, they will be on a final amplitude of $4 \mathrm{~V}$.

\section{Phase IV: Post-operative Follow-up Phase Post-operative Assessments, Aiming to Track the Neural and Symptomatic Change As a Result of DBS, Will Involve}

Weekly Self-report-rating scales of eating disorder psychopathology, anxiety and depression involving the True Colours text-messaging system started in phase I: http://oxtext.psych. ox.ac.uk/true-colours (5 min) (see Phase II: True Colours) (see Figure 1 for more details). Weekly side effects questionnaires, the SAFTEE-SI (81) and DBS side effects questionnaire (82).

Monthly

- Interview with Rebecca Park and Jessica Scaife. Repeat of baseline measures collected pre-operatively [see Phase II: Assessment with Research Team (Repeated at All Subsequent Monthly Follow-ups)].

- Joint neurosurgery-psychiatry reviews with Rebecca Park and Tipu Aziz, senior nurse practitioner and Jessica Scaife. During this review, neurosurgical parameters (such as the degree of stimulation) and psychiatric parameters (such as experience of eating disorder and co-morbid psychiatric symptoms) will be jointly assessed. Any necessary adjustments to stimulator intensity will then be programmed.

\section{Post-operative Neuroimaging}

- Resting state and food "wanting" task under MEG (13), at OHBA, at month 3 (pre-DBS switch-on), two scans in month 10 in DBS on and off conditions and at the end of study in month 15. Post-operative MRI is not possible with the stimulator implanted for safety reasons.

- Following each MEG scan, participants will carry out the LOFPQ food reward task. Tasks from the CANTAB (80) and a task measuring habit formation (50) will be carried out twice during the DBS on-off phase and at the end of the study (see Figure 1).

Post-operative neuropsychological assessment at 15 months (12 months after DBS switch-on) will be a repeat of those tests detailed in Section "Phase II: Neuropsychological Assessment."

\section{Phase V: Ethics Sub-Study}

The protocol for the ethics sub-study was recently published (52), giving best-practice guidelines for such research worldwide. A separate, optional post-operative ethics sub-study will also be offered. Participants will be informed about this option in the main study PIS. In the main study consent form, they will be asked if they agree to being contacted about the post-operative ethics sub-study.

It will involve one 2.5-hour session with an independent psychiatrist, Jacinta Tan, near the end of the post-operative follow-up period (after 6 months). The sub-study will have a separate PIS and separate informed consent form. This consent form will be requested for the participant's permission to draw upon the assessment recordings and measures obtained during the main DBS study for use in the subsequent ethical research and analysis.

If the patients wish to be contacted regarding this ethics sub-study, they will be sent the information sheet during the follow-up period. If they consent to participation, the single post-operative interview will occur at the end of the postoperative follow-up period, in order to maintain Jacinta Tan's roles as an advocate and a clinical researcher distinct.

The post-operative ethics interview will explore the ethical issues with the participant that surround the research. Jacinta Tan will elicit her experience of participation, her reflections on this, and also views in hindsight of the ethical issues surrounding the research from the participant.

At the end of the 15-month protocol period, the patient has the option to remove the DBS device which can be done in a simple operation with minimal risks. There will be the option for an annual research follow-up for up to 4 years. At the end of protocol period, or at any point subsequently, the patient has the option to remove the DBS device which can be done in a simple operation with minimal risks. If the participant decides to keep the DBS stimulator in situ, they will have routine neurosurgical DBS follow-up every 12 months for clinical care. The annual research follow-up if consented to would take place at the same time and include the following:

- The same questionnaires as completed monthly during the main study.

- The same computerised tasks as completed pre- and post operation.

- A FaceTime/Skype or phone interview covering psychiatric symptoms and individual experience.

- The same neuropsychological battery they completed pre-operatively.

\section{SAFETY}

An SAE is any untoward medical occurrence that results in death, is life-threatening, requires inpatient hospitalisation or prolongation of existing hospitalisation, or results in persistent or significant disability/incapacity.

\section{Reporting Procedures for SAEs}

"An SAE occurring to a participant should be reported to the REC that gave a favorable opinion of the study, where in the opinion 
of the Chief Investigator (CI) the event was: 'related' - that is, it resulted from administration of any of the research procedures; and 'unexpected' - that is, the type of event is not listed in the protocol as an expected occurrence. Reports of related and unexpected SAEs should be submitted within 15 days of the CI becoming aware of the event, using the NRES report of serious adverse event form (see IRAS/NRES website). All reports will be cc'd to the sponsor Clinical Trials \& Research Governance, University of Oxford (CTRG)".

\section{Data Analysis}

Number of participants: this is an exploratory study of up to six patients, and therefore a precise power calculation is not possible. External review of the protocol for an initial single patient pilot (by the international expert in the neuroscience of AN, Professor Kaye, University of San Diego) suggested the scientific importance of extending the study to include more patients.

Treatment effects will be established using within-subject analyses comparing baseline characteristics with patient assessment at each monthly follow-up session and by comparing change in symptoms during periods when the stimulator is "on" and periods when the stimulator is "off." We plan to use multiple regression, analysis of variance and $t$-tests using SPSS software to analyse the data. Results can be compared to our published findings from a linked multimodal neuroimaging case-control study of reward processing in current $\mathrm{AN}$, recovered $\mathrm{AN}$ and controls $(12,13,36)$.

In addition, MEG data will be analysed using in-house software and open software packages. Preprocessing of data will rely on propriety software supplied by the scanner manufacturer. We will employ both signal and source space methods, building on our previous approach to analysing and interpreting neural data obtained in patients with AN. Non-parametric statistics and general linear modelling will be used to establish significance.

\section{Data Monitoring}

Data monitoring for the project will be conducted by the CI and co-investigators. The study may also be audited by the CTRG. There will be no external monitoring of the study. The project will be under ongoing review for safety and efficacy by the CI and Dr. Park. The study may also be audited by the CTRG, with extensive protocols from the University of Oxford. Any SAE directly relating to the DBS electrode insertion and which cannot be immediately resolved will result in electively stopping the trial prematurely.

\section{PARTICIPANT CONFIDENTIALITY}

The study team will ensure that the participants' anonymity is maintained. A basic dataset of name, age, predominant symptoms and medications will be recorded. All electronic data will be anonymised with the date of recording and a unique project number as the only identifiers. It will be stored on secured university servers and is covered by the Oxford University Data protection register (reference No. Z575783X). All non-anonymised paper

${ }^{1}$ University of Oxford. Available: http://researchsupport.admin.ox.ac.uk/ctrg data (e.g., consent forms) will be stored in a locked cupboard within a secure building. All paper records will be kept in the research department at all times.

\section{Capacity and Informed Consent}

Ethical issues are of major importance in the treatment of $\mathrm{AN}$ and will be the focus of an ethical sub-study (detailed in section Phase V: Ethics Sub-Study) (52). Only patients with full capacity, fully consenting and voluntarily entering into the study will be eligible.

\section{Burdens}

The study does involve a number of return visits for outpatient assessment and follow-up, and includes up to 4 days spent in hospital for the DBS surgery in order for these assessments to be carried out. Therefore, we will aim to recruit patients who are physically mobile and are prepared and able to return for multiple assessments as outlined. However, involvement is entirely voluntary, and the patient can withdraw at any time.

\section{DISCUSSION}

We have presented an innovative clinical trial protocol, applying DBS research to individuals with SE-AN. We hope this will guide future researcher, ethics committees and the development of technologies designed for psychiatric populations. We recommend this protocol as a starting point to guide future trials. We further suggest that the neuroethical framework we have now published (52) should also be incorporated in such experimental treatment trials in SE-AN, due to unique concerns regarding physical wellness and capacity. The protocol we describe incorporates this ethical gold standard (52), which acts in synergy with the main trials protocol described here. It serves as means to investigate, empirically, the ethics of such an experimental treatment. Incorporating neuroethical standards centrally within novel neurotechnology research aims to ensure that rigorous and continuous ethical input is available. This is of great importance given the sensitive and challenging nature of this research, and it is essential that the welfare of vulnerable participants is maximally protected. Our protocol exemplifies the Nuffield Council of Bioethics' recommendations regarding the ethics of novel neurotechnology (2013) (79). We believe that the clinical trial described here can contribute to treatment development for $\mathrm{AN}$ and the ethics of such research in addition to extending the boundaries of DBS science.

\section{AVAILABILITY OF DATA AND MATERIALS}

The authors can confirm that all relevant data are included in the article. Data generated by the protocol will be included in subsequent publications and/or supplementary information files once the study is completed. Dissemination will be by presentation at conferences and in peer-reviewed publications.

\section{INSURANCE}

In the event of any participant suffering harm as a result of their involvement in the research, the University has a 
specialist insurance policy in place which would operate (Newline Underwriting Management Ltd., at Lloyd's of London, policy numbered: WD1200463).

\section{TRIAL STATUS}

The trial status is ongoing, and recruitment commenced on 8 October, 2013. Active recruitment had to be paused after the first patient due to MEG scanner refurbishment (4/15-4/16): protocol version 6, dated 11 November, 2016. To date, five participants have been enrolled in the study. The study has been approved by NRES Committee South Central-Oxford A REC (Ref: 13/SC/0267) and is registered at clinicaltrials.gov (NCT01924598).

\section{ETHICS STATEMENT}

Ethics approval and consent: this study will be carried out in accordance with the recommendations of NRES: South Central-Oxford A Research Ethics Committee (REC) Ref: 13/ SC/0267. Informed written consent will be taken care by RP, in accordance with the Declaration of Helsinki. Consent can be freely withdrawn at any point with no effect on their usual clinical care.

\section{REFERENCES}

1. Keel PK, Dorer DJ, Eddy KT, Franko D, Charatan DL, Herzog DB. Predictors of mortality in eating disorders. Arch Gen Psychiatry (2003) 60(2):179-83. doi:10.1001/archpsyc.60.2.179

2. Franko DL, Keshaviah A, Eddy KT, Krishna M, Davis MC, Keel PK, et al. A longitudinal investigation of mortality in anorexia nervosa and bulimia nervosa. Am J Psychiatry (2013) 170(8):917-25. doi:10.1176/appi.ajp.2013. 12070868

3. Steinhausen HC. The outcome of anorexia nervosa in the 20th century. Am J Psychiatry (2002) 159(8):1284-93. doi:10.1176/appi.ajp.159.8.1284

4. Bulik CM, Sullivan PF, Tozzi F, Furberg H, Lichtenstein P, Pedersen NL. Prevalence, heritability, and prospective risk factors for anorexia nervosa. Arch Gen Psychiatry (2006) 63(3):305-12. doi:10.1001/archpsyc.63.3.305

5. Watson H, Bulik C. Update on the treatment of anorexia nervosa: review of clinical trials, practice guidelines and emerging interventions. Psychol Med (2012) 10:1-24. doi:10.1017/S0033291712002620

6. Crow SJ, Mitchell JE, Roerig JD, Steffen K. What potential role is there for medication treatment in anorexia nervosa? Int J Eat Disord (2009) 42(1):1-8. doi:10.1002/eat.20576

7. Balestrieri M, Oriani MG, Simoncini A, Bellantuono C. Psychotropic drug treatment in anorexia nervosa. Search for differences in efficacy/tolerability between adolescent and mixed-age population. Eur Eat Disord Rev (2013) 21(5):361-73. doi:10.1002/erv.2240

8. Frank GK, Shott ME. The role of psychotropic medications in the management of anorexia nervosa: rationale, evidence and future prospects. CNS Drugs (2016) 30(5):419-42. doi:10.1007/s40263-016-0335-6

9. Park RJ, Godier LR, Cowdrey FA. Hungry for reward: how can neuroscience inform the development of treatment for anorexia nervosa? Behav Res Ther (2014) 62:47-59. doi:10.1016/j.brat.2014.07.007

10. Cowdrey FA, Filippini N, Park RJ, Smith SM, McCabe C. Increased resting state functional connectivity in the default mode network in recovered anorexia nervosa. Hum Brain Mapp (2012) 35(2):483-91. doi:10.1002/Hbm.22202

11. Gaudio S, Wiemerslage L, Brooks SJ, Schioth HB. A systematic review of resting-state functional-MRI studies in anorexia nervosa: evidence for functional connectivity impairment in cognitive control and visuospatial and

\section{AUTHOR CONTRIBUTIONS}

RP designed the study, wrote the protocol, information sheets, ethical applications and grant applications and led on all psychiatric aspects of the study, in liaison with TA who contributed all surgical detail and led on all surgical aspects of the study. RP and JS will acquire and analyse the data. RP and JS drafted the manuscript. All authors read and approved the final manuscript.

\section{ACKNOWLEDGMENTS}

The authors would like to acknowledge the clinical DBS team at the John Radcliffe Hospital for their support in this study and Alexandra Pike for her assistance in preparing the manuscript. The authors acknowledge Jacinta Tan for her contribution to the ethical sub-study.

\section{FUNDING}

Funding for the first case was provided by an MRC Confidence in Concept Award MC_PC_12020 awarded to RP. Further cases have been supported by grants from the Charles Wolfson Charitable Trust and the Swiss Anorexia Foundation. These funding bodies had no role in the design of the study, collection, analysis and interpretation of data or in writing the manuscript.

body-signal integration. Neurosci Biobehav Rev (2016) 71:578-89. doi:10.1016/ j.neubiorev.2016.09.032

12. Scaife J, Godier L, Filippini N, Harmer C, Park R. Reduced resting state functional connectivity in current and recovered restrictive anorexia nervosa. Front Psychiatry (2017) 8:30. doi:10.3389/fpsyt.2017.00030

13. Godier LR, Scaife JC, Braeutigam S, Park RJ. Enhanced early neuronal processing of food pictures in anorexia nervosa: a magnetoencephalography study. Psychiatry J (2016) 2016:1795901. doi:10.1155/2016/1795901

14. Lozano AM, Mayberg HS, Giacobbe P, Hamani C, Craddock RC, Kennedy SH. Subcallosal cingulate gyrus deep brain stimulation for treatment-resistant depression. Biol Psychiatry (2008) 64(6):461-7. doi:10.1016/j.biopsych.2008. 05.034

15. BewernickBH, Hurlemann R, Matusch A, Kayser S, Grubert C, Hadrysiewicz B, et al. Nucleus accumbens deep brain stimulation decreases ratings of depression and anxiety in treatment-resistant depression. Biol Psychiatry (2010) 67(2):110-6. doi:10.1016/j.biopsych.2009.09.013

16. Lozano AM, Giacobbe P, Hamani C, Rizvi SJ, Kennedy SH, Kolivakis TT, et al. A multicenter pilot study of subcallosal cingulate area deep brain stimulation for treatment-resistant depression. J Neurosurg (2012) 116(2):315-22. doi:10.3171/2011.10.JNS102122

17. Bergfeld IO, Mantione M, Hoogendoorn ML, Ruhe HG, Horst F, Notten P, et al. Impact of deep brain stimulation of the ventral anterior limb of the internal capsule on cognition in depression. Psychol Med (2017) 47:1647-58. doi:10.1017/S0033291717000113

18. Lipsman N, Neimat JS, Lozano AM. Deep brain stimulation for treatmentrefractory obsessive-compulsive disorder: the search for a valid target. Neurosurgery (2007) 61(1):1-11; discussion 11-3. doi:10.1227/01.neu.0000279719. 75403.f7

19. de Haan S, Rietveld E, Stokhof M, Denys D. Becoming more oneself? Changes in personality following DBS treatment for psychiatric disorders: experiences of OCD patients and general considerations. PLoS One (2017) 12(4):e0175748. doi:10.1371/journal.pone.0175748

20. Velasques B, Diniz C, Teixeira S, Cartier C, Peressutti C, Silva F, et al. Deep brain stimulation: a new treatment in mood and anxiety disorders. CNS Neurol Disord Drug Targets (2014) 13(6):961-71. doi:10.2174/18715273136 66140612122929 
21. Greenberg BD, Gabriels LA, Malone DA Jr, Rezai AR, Friehs GM, Okun MS, et al. Deep brain stimulation of the ventral internal capsule/ventral striatum for obsessive-compulsive disorder: worldwide experience. Mol Psychiatry (2010) 15(1):64-79. doi:10.1038/mp.2008.55

22. Halmi KA, Eckert E, Marchi P, Sampugnaro V, Apple R, Cohen J. Comorbidity of psychiatric diagnoses in anorexia nervosa. Arch Gen Psychiatry (1991) 48(8):712-8. doi:10.1001/archpsyc.1991.01810320036006

23. Godier LR, Park RJ. Compulsivity in anorexia nervosa: a transdiagnostic concept. Front Psychol (2014) 5:778. doi:10.3389/fpsyg.2014.00778

24. Godier LR, Park RJ. Does compulsive behavior in anorexia nervosa resemble an addiction? A qualitative investigation. Front Psychol (2015) 6:1608. doi:10.3389/fpsyg.2015.01608

25. McLaughlin NC, Didie ER, Machado AG, Haber SN, Eskandar EN, Greenberg BD. Improvements in anorexia symptoms after deep brain stimulation for intractable obsessive-compulsive disorder. Biol Psychiatry (2013) 73(9):e29-31. doi:10.1016/j.biopsych.2012.09.015

26. Israel M, Steiger H, Kolivakis T, McGregor L, Sadikot AF. Deep brain stimulation in the subgenual cingulate cortex for an intractable eating disorder. Biol Psychiatry (2010) 67(9):e53-4. doi:10.1016/j.biopsych.2009. 11.016

27. Wu H, Van Dyck-Lippens PJ, Santegoeds R, van Kuyck K, Gabriels L, Lin G, et al. Deep-brain stimulation for anorexia nervosa. World Neurosurg (2013) 80(3-4):e21-10. doi:10.1016/j.wneu.2012.06.039

28. Blomstedt P, Naesström M, Bodlund O. Deep brain stimulation in the bed nucleus of the stria terminalis and medial forebrain bundle in a patient with major depressive disorder and anorexia nervosa. Clin Case Rep (2017) 5:679-84. doi:10.1002/ccr3.856

29. Lipsman N, Woodside DB, Giacobbe P, Hamani C, Carter JC, Norwood SJ, et al. Subcallosal cingulate deep brain stimulation for treatment-refractory anorexia nervosa: a phase 1 pilot trial. Lancet (2013) 381(9875):1361-70. doi:10.1016/S0140-6736(12)62188-6

30. Lipsman N, Lam E, Volpini M, Sutandar K, Twose R, Giacobbe P, et al. Deep brain stimulation of the subcallosal cingulate for treatment-refractory anorexia nervosa: 1 year follow-up of an open-label trial. Lancet Psychiatry (2017) 4(4):285-94. doi:10.1016/S2215-0366(17)30076-7

31. Park RJ, Dunn BD, Barnard PJ. Schematic models and modes of mind in anorexia nervosa I: a novel process account. Int J Cogn Ther (2011) 4(4): 415-37. doi:10.1521/ijct.2011.4.4.415

32. Park RJ, Dunn BD, Barnard PJ. Schematic models and modes of mind in anorexia nervosa II: implications for treatment and course. Int J Cogn Ther (2012) 5(1):86-98. doi:10.1521/ijct.2012.5.1.86

33. Fladung AK, Gron G, Grammer K, Herrnberger B, Schilly E, Grasteit S, et al. A neural signature of anorexia nervosa in the ventral striatal reward system. Am J Psychiatry (2010) 167(2):206-12. doi:10.1176/appi.ajp.2009. 09010071

34. Fladung AK, Schulze UM, Scholl F, Bauer K, Gron G. Role of the ventral striatum in developing anorexia nervosa. Transl Psychiatry (2013) 3:e315. doi:10.1038/tp. 2013.88

35. Cowdrey FA, Finlayson G, Park RJ. Liking compared with wanting for high- and low-calorie foods in anorexia nervosa: aberrant food reward even after weight restoration. Am J Clin Nutr (2013) 97(3):463-70. doi:10.3945/ ajcn.112.046011

36. Scaife JC, Godier LR, Reinecke A, Harmer CJ, Park RJ. Differential activation of the frontal pole to high vs low calorie foods: the neural basis of food preference in anorexia nervosa? Psychiatry Res (2016) 258:44-53. doi:10.1016/j. pscychresns.2016.10.004

37. Wagner A, Aizenstein H, Venkatraman VK, Bischoff-Grethe A, Fudge J, May JC, et al. Altered striatal response to reward in bulimia nervosa after recovery. Int J Eat Disord (2010) 43(4):289-94. doi:10.1002/eat.20699

38. Berridge KC, Kringelbach ML. Pleasure systems in the brain. Neuron (2015) 86(3):646-64. doi:10.1016/j.neuron.2015.02.018

39. van der Plasse G, Schrama R, van Seters SP, Vanderschuren LJ, Westenberg HG. Deep brain stimulation reveals a dissociation of consummatory and motivated behaviour in the medial and lateral nucleus accumbens shell of the rat. PLoS One (2012) 7(3):e33455. doi:10.1371/journal.pone.0033455

40. Hill JO, Berridge K, Avena NM, Ziauddeen H, Alonso-Alonso M, Allison DB, et al. Neurocognition: the food-brain connection. Adv Nutr (2014) 5(5):544-6. doi:10.3945/an.114.006437
41. Kaye WH, Wierenga CE, Bailer UF, Simmons AN, Bischoff-Grethe A. Nothing tastes as good as skinny feels: the neurobiology of anorexia nervosa. Trends Neurosci (2013) 36(2):110-20. doi:10.1016/j.tins.2013.01.003

42. Kaye WH, Wierenga CE, Bailer UF, Simmons AN, Wagner A, BischoffGrethe A. Does a shared neurobiology for foods and drugs of abuse contribute to extremes of food ingestion in anorexia and bulimia nervosa? Biol Psychiatry (2013) 73(9):836-42. doi:10.1016/j.biopsych.2013. 01.002

43. Oudijn MS, Storosum JG, Nelis E, Denys D. Is deep brain stimulation a treatment option for anorexia nervosa? BMC Psychiatry (2013) 13:277. doi:10.1186/1471-244X-13-277

44. Walsh BT. The enigmatic persistence of anorexia nervosa. Am J Psychiatry (2013) 170(5):477-84. doi:10.1176/appi.ajp.2012.12081074

45. Lochner C, Fineberg NA, Zohar J, van Ameringen M, Juven-Wetzler A, Altamura AC, et al. Comorbidity in obsessive-compulsive disorder (OCD): a report from the International College of Obsessive-Compulsive Spectrum Disorders (ICOCS). Compr Psychiatry (2014) 55(7):1513-9. doi:10.1016/j. comppsych.2014.05.020

46. Robbins TW, Everitt BJ. Neurobehavioural mechanisms of reward and motivation. Curr Opin Neurobiol (1996) 6(2):228-36. doi:10.1016/S09594388(96)80077-8

47. Steinglass J, Walsh BT. Habit learning and anorexia nervosa: a cognitive neuroscience hypothesis. Int J Eat Disord (2006) 39(4):267-75. doi:10.1002/ eat. 20244

48. Godier LR, de Wit S, Pinto A, Steinglass JE, Greene AL, Scaife J, et al. An investigation of habit learning in anorexia nervosa. Psychiatry Res (2016) 244:214-22. doi:10.1016/j.psychres.2016.07.051

49. Gillan CM, Kosinski M, Whelan R, Phelps EA, Daw ND. Characterizing a psychiatric symptom dimension related to deficits in goal-directed control. Elife (2016) 5. doi:10.7554/eLife.11305

50. Gillan CM, Otto AR, Phelps EA, Daw ND. Model-based learning protects against forming habits. Cogn Affect Behav Neurosci (2015) 15(3):523-36. doi:10.3758/s13415-015-0347-6

51. Arcelus J, Mitchell AJ, Wales J, Nielsen S. Mortality rates in patients with anorexia nervosa and other eating disorders. A meta-analysis of 36 studies. Arch Gen Psychiatry (2011) 68(7):724-31. doi:10.1001/archgenpsychiatry. 2011.74

52. Park RJ, Singh I, Pike AC, Tan JOA. Deep brain stimulation in anorexia nervosa: hope for the hopeless or exploitation of the vulnerable? The Oxford neuroethics gold standard framework. Front Psychiatry (2017) 8:44. doi:10.3389/ fpsyt.2017.00044

53. Denys D, Mantione M, Figee $M$, van den Munckhof P, Koerselman F, Westenberg $\mathrm{H}$, et al. Deep brain stimulation of the nucleus accumbens for treatment-refractory obsessive-compulsive disorder. Arch Gen Psychiatry (2010) 67(10):1061-8. doi:10.1001/archgenpsychiatry.2010.122

54. Fairburn CG, Cooper Z, O’Connor M. Eating Disorder Examination (edition 16.0D). Cognitive Behaviour Therapy and Eating Disorders. New York: Guilford Press (2008).

55. American Psychiatric Association. Diagnostic and Statistical Manual of Mental Disorders. Washington, DC: American Psychiatric Association (2000).

56. American Psychiatric Association. Diagnostic and Statistical Manual of Mental Disorders. 5th ed. Arlington, VA: American Psychiatric Publishing (2013).

57. Hamilton M. The assessment of anxiety states by rating. Br J Med Psychol (1959) 32(1):50-5. doi:10.1111/j.2044-8341.1959.tb00467.x

58. Hamilton M. A rating scale for depression. J Neurol Neurosurg Psychiatry (1960) 23:56-62. doi:10.1136/jnnp.23.1.56

59. Goodman WK, Price LH, Rasmussen SA, Mazure C, Fleischmann RL, Hill CL, et al. The Yale-Brown Obsessive Compulsive Scale. I. Development, use, and reliability. Arch Gen Psychiatry (1989) 46(11):1006-11. doi:10.1001/ archpsyc.1989.01810110048007

60. Mazure CM, Halmi KA, Sunday SR, Romano SJ, Einhorn AM. The YaleBrown-Cornell Eating Disorder Scale: development, use, reliability and validity. J Psychiatr Res (1994) 28(5):425-45. doi:10.1016/0022-3956(94) 90002-7

61. Fairburn CG, Beglin S. Eating Disorder Examination Questionnaire (EDE-Q 6.0). In: Fairburn C, editor. Cognitive Behaviour Therapy and Eating Disorders. New York: Guilford Press (2008). p. 309-13. 
62. Godier LR, Park RJ. A novel measure of compulsive food restriction in anorexia nervosa: validation of the Self-Starvation Scale (SS). Eat Behav (2015) 17:10-3. doi:10.1016/j.eatbeh.2014.12.004

63. Bohn K, Doll HA, Cooper Z, O'Connor M, Palmer RL, Fairburn CG. The measurement of impairment due to eating disorder psychopathology. Behav Res Ther (2008) 46(10):1105-10. doi:10.1016/j.brat.2008.06.012

64. Skevington SM. Measuring quality of life in Britain: introducing the WHOQOL-100. J Psychosom Res (1999) 47(5):449-59. doi:10.1016/S00223999(99)00051-3

65. Beck AT, Steer RA, Brown GK. Manual for the Beck Depression Inventory-II. San Antonio, TX: Psychological Corporation (1996).

66. Spielberger CD. Manual for the State-Trait Anxiety Inventory (Form Y). Palo Alto, CA: Mind Garden (1983).

67. Snaith RP, Hamilton M, Morley S, Humayan A, Hargreaves D, Trigwell P. A scale for the assessment of hedonic tone the Snaith-Hamilton Pleasure Scale. Br J Psychiatry (1995) 167(1):99-103. doi:10.1192/bjp.167.1.99

68. Cowdrey FA, Park RJ. Assessing rumination in eating disorders: principal component analysis of a minimally modified ruminative response scale. Eat Behav (2011) 12(4):321-4. doi:10.1016/j.eatbeh.2011.08.001

69. Wechsler D. Wechsler Adult Intelligence Scale. 4th ed. San Antonio, TX: Pearson (2008).

70. Coughlan AK, Oddy M, Crawford AR. BIRT Memory and Information Processing Battery (BMIPB). London: Brain Injury Rehabilitation Trust (2007).

71. Spreen O,Strauss E. A Compendium of Neuropsychological Tests: Administration, Norms and Commentary. New York: Oxford University Press (1998).

72. Meyers JE, Meyers KR. Rey Complex Figure (RCF) Task. Lutz, USA: PAR (1995).

73. Bechara A. Iowa Gambling Task: Professional Manual. Lutz, Florida, USA: PAR (2007).

74. Strauss E, Sherman EMS, Spreen O. A Compendium of Neuropsychological Tests. 3rd ed. New York, USA: Oxford University Press (2006).

75. Delis DC, Kaplan E, Kramer JH. Delis Kaplan Executive Function System. San Antonio, USA: The Psychological Corporation (2001).
76. Zigmund AS, Snaith RP. The hospital anxiety and depression scale. Acta Psychiatr Scand (1983) 67:361-70. doi:10.1111/j.1600-0447.1983. tb09716.x

77. Appelbaum PS, Grisso T. MacArthur Competence Assessment Tool for Clinical Research (MacCAT-CR). Sarasota, FL: Professional Resource Press (2001).

78. Nuttin B, Wu H, Mayberg H, Hariz M, Gabriels L, Galert T, et al. Consensus on guidelines for stereotactic neurosurgery for psychiatric disorders. J Neurol Neurosurg Psychiatry (2014) 85(9):1003-8. doi:10.1136/jnnp-2013-306580

79. Nuffield Council on Bioethics. Novel Neurotechnologies: Intervening in the Brain. London: Nuffield Council on Bioethics (2013).

80. CANTAB ${ }^{\circledast}$ [Cognitive Assessment Software]. Cambridge Cognition (2017). Available from: www.cantab.com

81. Clyde DJ. SAFTEE: data system for side effect assessment scale. Psychopharmacol Bull (1986) 22(1):287.

82. Brunoni AR, Amadera J, Berbel B, Volz MS, Rizzerio BG, Fregni F. A systematic review on reporting and assessment of adverse effects associated with transcranial direct current stimulation. Int J Neuropsychopharmacol (2011) 14(8):1133-45. doi:10.1017/S1461145710001690

Conflict of Interest Statement: The patients will experience excellent follow-up quality clinical care from the neurosurgical DBS team irrespective of their trial involvement. The CI on the study is not responsible for the ongoing clinical psychiatric care of the patient, and therefore conflicts of interest should not arise. Involvement in this trial will not affect the choice of operation or longer-term stimulation parameters. There are no commercial conflicts of interest as there is no financial gain for researchers or patients.

Copyright (c) 2018 Park, Scaife and Aziz. This is an open-access article distributed under the terms of the Creative Commons Attribution License (CC BY). The use, distribution or reproduction in other forums is permitted, provided the original author(s) and the copyright owner are credited and that the original publication in this journal is cited, in accordance with accepted academic practice. No use, distribution or reproduction is permitted which does not comply with these terms. 